\title{
Prevalência e aspectos epidemiológicos da coinfecção da tuberculose e HIV no Estado
}

\section{do Tocantins, 2001-2020}

Prevalence and epidemiological aspects of tuberculosis and hiv coinfection in the State of Tocantins, 2001-2020

\section{Prevalencia y aspectos epidemiológicos de la coinfección de tuberculosis y VIH en el Estado de}

Tocantins, 2001-2020

\author{
Millena Horner Nogueira \\ ORCID: https://orcid.org/0000-0001-6626-1623 \\ Instituto Tocantinense Presidente Antônio Carlos, Brasil \\ E-mail: millena_horner@outlook.com \\ Carolinne Lisboa Silva \\ ORCID: https://orcid.org/0000-0002-9305-562X \\ Instituto Tocantinense Presidente Antônio Carlos, Brasil \\ E-mail: calisboa94@gmail.com \\ Samuel Henrique Silva Souza \\ ORCID: https://orcid.org/0000-0001-7782-1948 \\ Instituto Tocantinense Presidente Antônio Carlos, Brasil \\ E-mail: samuelsouza243@gmail.com \\ Laís Freitas Soares \\ ORCID: https://orcid.org/0000-0001-7415-502X \\ Instituto Tocantinense Presidente Antônio Carlos, Brasil \\ E-mail: lais.fsoares@hotmail.com \\ Lorena Dias Monteiro \\ ORCID: https://orcid.org/0000-0002-2246-3490 \\ Instituto Tocantinense Presidente Antônio Carlos, Brasil \\ E-mail: lorenamonteiro3@hotmail.com
}

\begin{abstract}
Resumo
Objetivo: Descrever a prevalência e o perfil epidemiológico da coinfecção tuberculose e o vírus HIV no estado do Tocantins. Metodologia: Estudo epidemiológico descritivo de casos novos de tuberculose coinfectados com HIV registrados no período de 2001 a 2020 no Tocantins. Os dados foram coletados do Departamento de Informática do Sistema Único de Saúde (DATASUS) e tabulados em gráficos e tabelas. Resultados: Foram registrados 199 casos novos de tuberculose coinfectados por HIV, prevalência de 5\%. Houve aumento da coinfecção de tuberculose e HIV de 2,75\% em 2001 para 8,90\% em 2020. Predominaram os casos do sexo masculino (76,39\%), adultos jovens $(50,80 \%)$, pardos $(68,87 \%)$ e predominância na região de saúde Capim Dourado $(36,20 \%)$. A forma clínica extrapulmonar representou $34,17 \%$. O percentual de cura foi de apenas 45,22\%. Conclusão: O aumento de casos de tuberculose coinfectados com HIV, a gravidade dos casos na forma clínica extrapulmonar, o baixo percentual de cura e alta carga da letalidade representam os desafios que os programas de controle precisam superar para o manejo clínico da tuberculose associada ao HIV. O principal desafio inclui a integração de um tratamento eficaz de tuberculose associado ao uso de terapia antirretroviral para a prevenção de comorbidades relacionadas ao HIV.
\end{abstract}

Palavras-chave: Tuberculose; Infecções por HIV; Coinfecção; Epidemiologia.

\begin{abstract}
Objective: Describe the prevalence and epidemiological profile of the co-infection of tuberculosis and the HIV virus in the state of Tocantins. Method: Descriptive epidemiological study of new tuberculosis cases coinfected with HIV registered from 2001 to 2020 in Tocantins. Data were collected from the Information Technology Department of the Unified Health System (DATASUS) and tabulated into graphs and tables. Results: There were 199 new cases of TB coinfected with HIV, prevalence of 5\%. There was an increase in the co-infection of tuberculosis and HIV from $2.75 \%$ in 2001 to $8.90 \%$ in 2020 . Male cases predominated (76.39\%), young adults (50.80\%), browns $(68.87 \%)$ and predominance in the Capim Dourado health region (36.20\%). The extrapulmonary clinical form represented $34.17 \%$. The percentage of cure was only $45.22 \%$. Conclusion: The increase in HIV-coinfected tuberculosis cases, the severity of cases in the extrapulmonary clinical form, the low percentage of cures and the high mortality rate represent the challenges that control programs need to overcome for the clinical management of HIV-associated tuberculosis. The
\end{abstract}


main challenge includes the integration of effective tuberculosis treatment associated with the use of antiretroviral therapy for the prevention of HIV-related comorbidities.

Keywords: Tuberculosis; HIV infections; Coinfection; Epidemiology.

\begin{abstract}
Resumen
Objetivo: Describir la prevalencia y el perfil epidemiológico de la coinfección de la tuberculosis y el virus del VIH en el estado de Tocantins. Método: Estudio epidemiológico descriptivo de nuevos casos de tuberculosis coinfectados con VIH registrados entre 2001 y 2020 en Tocantins. Los datos se obtuvieron del Departamento de Tecnología de la Información del Sistema Único de Salud (DATASUS) y se tabularon en gráficos y tablas. Resultados: Hubo 199 nuevos casos de tuberculosis coinfectada con VIH, prevalencia del 5\%. Hubo un aumento de la coinfección de tuberculosis y VIH del 2,75\% en 2001 al 8,90\% en 2020. Predominaron los casos de hombres (76,39\%), adultos jóvenes $(50,80 \%)$, marrones $(68,87 \%)$ y predominio en el Capim Dourado. región sanitaria (36,20\%). La forma clínica extrapulmonar representó el 34,17\%. El porcentaje de curación fue solo del 45,22\%. Conclusión: El aumento de casos de tuberculosis coinfectados por VIH, la gravedad de los casos en la forma clínica extrapulmonar, el bajo porcentaje de curas y la alta carga de mortalidad representan los desafíos que deben superar los programas de control para el manejo clínico de la tuberculosis asociada al VIH. El principal desafío incluye la integración de un tratamiento eficaz contra la tuberculosis asociado con el uso de la terapia antirretroviral para la prevención de las comorbilidades relacionadas con el VIH.
\end{abstract}

Palabras clave: Tuberculosis; Infecciones por VIH; Coinfección; Epidemiología.

\title{
1. Introdução
}

Embora se tenha alcançado melhorias significativas no diagnóstico da coinfecção do vírus da imunodeficiência humana (HIV) em casos de tuberculose (TB) nas últimas décadas, a doença persiste como problema de saúde pública em vários países do mundo, incluindo o Brasil. As pessoas vivendo com HIV estão mais propensas a desenvolver TB. Estima-se que essas pessoas tenham entre 16 e 27 vezes mais chances de evoluir para a forma ativa da doença, quando comparadas à população geral, elevando assim a taxa de complicações e mortalidade (Aaron \& Saadoun, 2004; Chakaya, 2020).

No mundo, cerca de 10 milhões de pessoas adoeceram com TB em 2019, destas, 8,2\% viviam com HIV. Além disso, 1,4 milhão de pessoas foram a óbito como consequência da TB, incluindo 208.000 pessoas coinfectadas com HIV. No Brasil, em 2019 a coinfecção de HIV foi de 8.070 casos registrados dentre os 78.428 novos casos de TB (Brasil, 2020; Chakaya, 2020).

A TB e o HIV constituem a principal carga de doenças infecciosas em países pobres. Nesses territórios, as coinfecções por HIV representam desafios diagnósticos e terapêuticos específicos e exercem uma pressão imensa sobre os sistemas de saúde com grandes populações de pessoas em situação de vulnerabilidade coinfectadas (Cavalin, 2020; Brasil, 2020; Ledesma, 2021; Ribas, 2021). Nessas pessoas, os dois patógenos, Mycobacterium tuberculosis e o vírus HIV, potencializam-se mutuamente, acelerando a deterioração das funções imunológicas e resultando em morte prematura se não tratados (Chakaya, 2020; Ledesma, 2021).

Embora a TB e a coinfecção por HIV sejam reconhecidas como um grande problema de saúde pública nos diferentes estados brasileiros, a sua magnitude é pouco conhecida pelas dificuldades que os programas de vigilância epidemiológica enfrentam na execução das ações de controle (Cavalin, 2020). Mesmo sendo uma doença com diagnóstico e tratamento realizados de forma universal e gratuita pelo Sistema Único de Saúde, ainda há barreiras no acesso (Cavalin, 2020; Ribas, 2021).

No Brasil, apesar do progresso na redução da morbidade e mortalidade por TB, os casos novos de TB com testagem para HIV aumentaram consideravelmente e o Tocantins é o $7^{\circ}$ estado com maior proporção de testagem (Brasil, 2020). Estudo recente realizado no Tocantins, identificou tendência de aumento significativo acima de $20 \%$ no coeficiente de incidência de TB em populações em situação de vulnerabilidade (Ribas, 2021). Diante essa carga crescente de TB em populações 
vulneráveis, esse estudo tem por objetivo descrever a prevalência e aspectos epidemiológicos da tuberculose e a coinfecção por HIV no estado do Tocantins no período de 2001 a 2020.

\section{Metodologia}

Realizou-se estudo epidemiológico descritivo com base em dados secundários sobre tuberculose e coinfecção de HIV na população maior de 13 anos de idade residentes no estado do Tocantins no período de 2001 a 2020, totalizando 199 casos novos. A coinfecção de HIV foi definida como a presença concomitante de TB e HIV / AIDS na mesma notificação. A epidemiologia descritiva examina como a incidência (casos novos) ou a prevalência (casos existentes) de uma doença ou condição relacionada à saúde varia de acordo com determinadas características, como sexo, idade, escolaridade e renda, entre outras (Costa \& Barreto, 2003).

Os dados analisados são provenientes de notificações compulsórias geradas nos serviços de saúde e estão disponíveis para domínio público no sítio eletrônico do DATASUS (BRASIL, 2019). A notificação compulsória é um documento padronizado com preenchimento sob responsabilidade do profissional de saúde em todo o país (BRASIL, 2016). Ademais, a Tuberculose está na Lista Nacional de Notificação Compulsória de doenças, agravos e eventos de saúde pública nos serviços de saúde públicos e privados em todo o território nacional como doença de notificação compulsória semanal (BRASIL, 2020).

Os dados foram coletados a partir do link http://tabnet.datasus.gov.br/cgi/tabcgi.exe?sinannet/cnv/tubercto.def do Departamento de Informática do Sistema Único de Saúde do Brasil, provenientes do Sistema de Informação de Agravos de Notificação - SINAN - do Ministério da Saúde (DATASUS/SINAN/MS) (Brasil, 2021). Tais dados são de notificação compulsória e foram preenchidos por profissionais da área de saúde, consistindo em dados padronizados com informações clínicas e sociodemográficas. Segundo Lakatos e Marconi (2001), a pesquisa documental é a coleta de dados em fontes primárias, como documentos escritos ou não, pertencentes a arquivos públicos; arquivos particulares de instituições e domicílios, e fontes estatísticas.

$\mathrm{Na}$ análise descritiva, as variáveis incluídas no estudo foram sexo, faixa etária, etnia, escolaridade, regiões de saúde, forma clínica da doença, bem como, as situações de encerramento foram exportadas e tabuladas para o Microsoft Excel@. Segundo Gil (1999), as pesquisas descritivas têm como finalidade principal a descrição das características de determinada população ou fenômeno, ou o estabelecimento de relações entre variáveis. São inúmeros os estudos que podem ser classificados sob este título e uma de suas características mais significativas aparece na utilização de técnicas padronizadas de coleta de dados.

A natureza da pesquisa quantitativa determina análise e estatística dos dados aqui analisados. Nesse sentido, segundo Richardson (1999), a pesquisa quantitativa é caracterizada pelo emprego da quantificação, tanto nas modalidades de coleta de informações quanto no tratamento delas por meio de técnicas estatísticas.

A tendência da proporção de coinfecção de HIV em casos novos de tuberculose foi calculada por meio da divisão entre o número de casos total de cada ano e o número total de casos multiplicados por 100 para delimitar a porcentagem. A análise do cálculo dos indicadores e elaboração das tabelas e figuras foram feitas em planilhas do Microsoft Excel.

Este estudo é baseado em dados secundários, e todas as informações apresentadas são de domínio público. Nenhuma variável permitiu a identificação de indivíduos. Assim, não foi necessária a aprovação do estudo por um Comitê de Ética em Pesquisa.

$\mathrm{O}$ estado de Tocantins, de onde foram coletados os dados, teve uma criação relativamente recente, quando em 5 de outubro de 1988 foi desmembrado do estado de Goiás, compreendendo um espaço geográfico de 277.423,630 km² (IBGE, 
2020), sendo subdividido, devido à sua extensão, em duas macrorregiões e em 8 regiões de saúde, com uma população estimada, em 2019 pelo IBGE, de 1.572.866 de habitantes, conforme demonstrado na Figura 1 e na Tabela 1 (IBGE, 2020).

Figura 1 - Regiões de saúde do Estado do Tocantins.

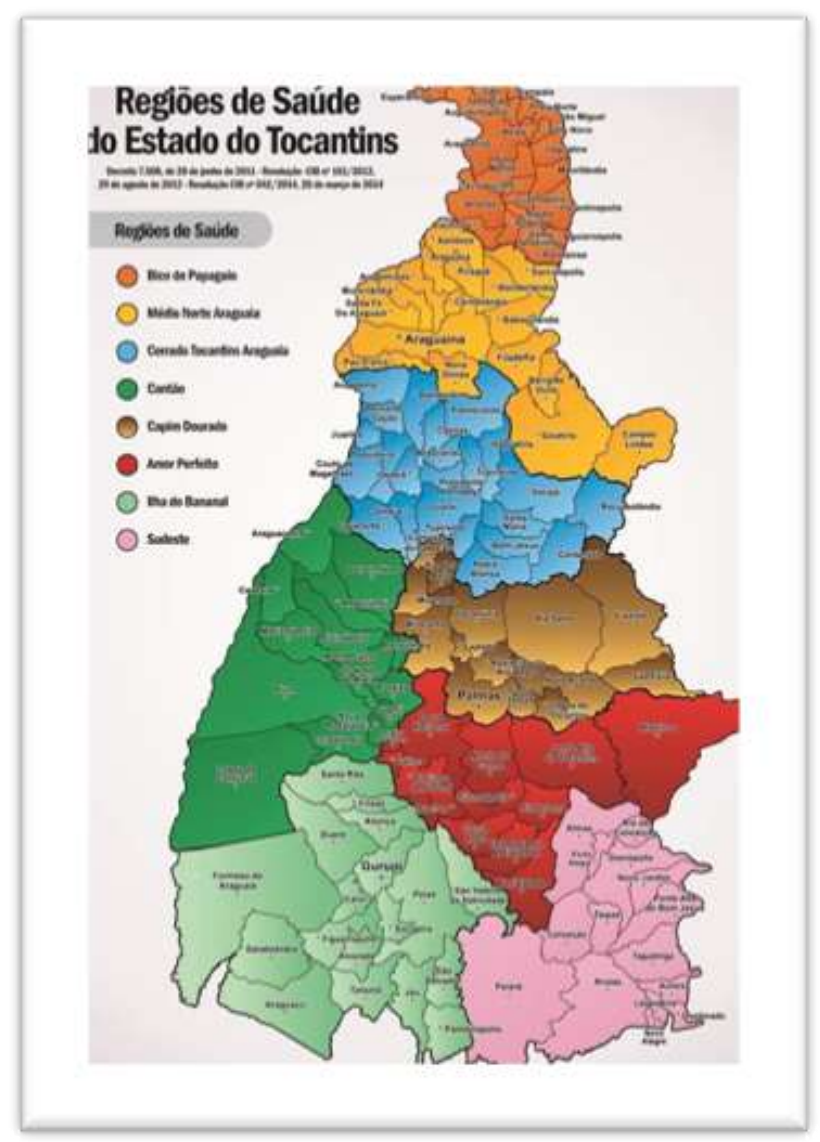

Fonte: Secretária de Saúde (2018).

Tabela 1 - Estimativa populacional abrangida nas regiões de saúde do Estado do Tocantins.

\begin{tabular}{|c|c|c|c|c|c|c|}
\hline $\begin{array}{l}\text { Região de } \\
\text { Saúde }\end{array}$ & Macrorregião & $\begin{array}{l}\text { Quant. De } \\
\text { Municípios }\end{array}$ & $\begin{array}{c}\text { População } \\
\text { (DATASUS 2001) }\end{array}$ & $\begin{array}{c}\text { População } \\
\text { (IBGE censo } \\
\text { 2010) } \\
\end{array}$ & $\begin{array}{c}\text { Populaçãa } \\
\text { (IBGE 2019) } \\
* \\
\end{array}$ & $\begin{array}{l}\text { \% Pop } \\
\text { Do } \\
\text { Estado }\end{array}$ \\
\hline Capim Dourado & \multirow{7}{*}{$\begin{array}{c}\text { Macrorregião } \\
\text { Centro Sul }\end{array}$} & 14 & 223.390 & 301.576 & 375.033 & $24 \%$ \\
\hline Ilha do Bananal & & 18 & 159.602 & 171.546 & 184.257 & $12 \%$ \\
\hline Cantão & & 15 & 97.761 & 114.648 & 130.124 & $8 \%$ \\
\hline Amor Perfeito & & 13 & 94.714 & 103.350 & 111.395 & $7 \%$ \\
\hline Sudeste & & 15 & 86.333 & 92.376 & 98.597 & $6 \%$ \\
\hline Soma/ Média & & 75 & 661.800 & 783.496 & 899.406 & $57 \%$ \\
\hline Médio Norte & & 17 & 220.256 & 262.650 & 301.862 & $19 \%$ \\
\hline Araguaia & \multirow{6}{*}{$\begin{array}{l}\text { Macrorregião } \\
\text { Norte }\end{array}$} & & & & & \\
\hline $\begin{array}{l}\text { Bico do } \\
\text { Panagaio }\end{array}$ & & 24 & 173.634 & 191.094 & 209.796 & $13 \%$ \\
\hline Cerrado & & 23 & 129.205 & 146.205 & 161.802 & $10 \%$ \\
\hline $\begin{array}{l}\text { Tocantins } \\
\text { Araguaia }\end{array}$ & & & & & & \\
\hline Soma/ Média & & 64 & 523.095 & 599.949 & 673.460 & $43 \%$ \\
\hline Total & & 139 & 1.184 .895 & 1.383 .445 & 1.572 .866 & $100 \%$ \\
\hline
\end{tabular}

Fonte: IBGE (2020). 
Na Figura 1 e Tabela 1 acima elencadas é possível identificar que na Macrorregião Centro Sul localiza-se a região de saúde Capim Dourado, identificada como a mais populosa com $24 \%$ do contingente populacional de todo o Estado, isso se deve a localização da Capital, Palmas.

\section{Resultados}

Foram notificados 3.975 casos novos de Tuberculose no período de 2001 a 2020, desses, 199 (5\%) estavam coinfectados por HIV. Apesar da flutuação na tendência da proporção de casos diagnosticados com a coinfecção de HIV nos 20 anos de avaliação, houve crescimento desse indicador (Figura 2).

Figura 2 - Prevalência de casos novos de tuberculose com coinfecção HIV residentes no estado do Tocantins, Brasil, nos anos de 2001 a 2020.

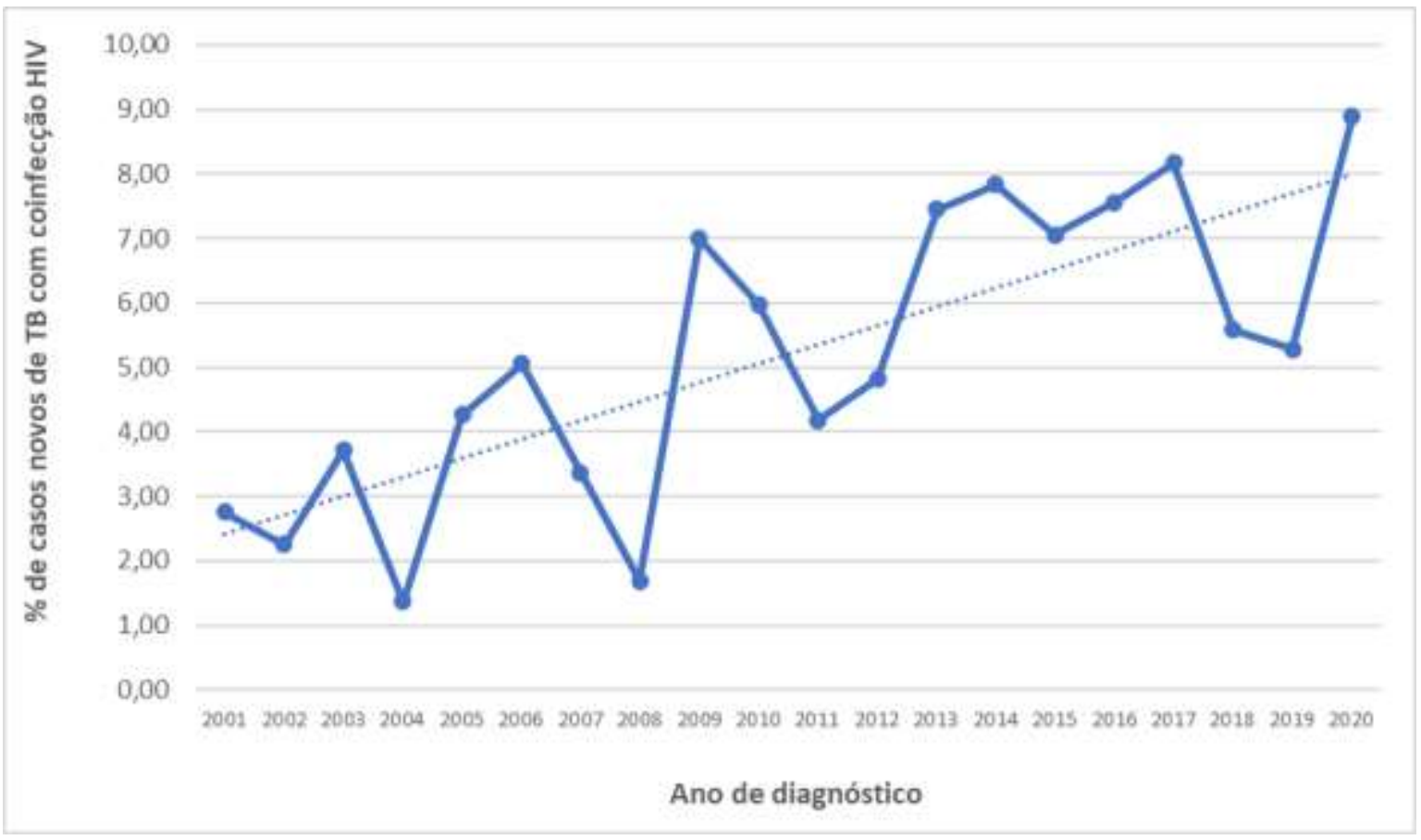

Fonte: Autores (2021).

Assim, é possível identificar que a reta pontilhada evidencia o crescimento da porcentagem de casos novos de TB com coinfecção HIV saltando de um pouco mais que 2\% em 2001 para quase 9\% em 2020.

A Tabela 2 apresenta a frequência de testagem sorológica dos casos de tuberculose para HIV de pessoas residentes no estado do Tocantins nos anos de 2001 a 2020. 
Tabela 2 - Frequência de testagem para HIV entre os casos novos de tuberculose residentes no estado do Tocantins, Brasil, nos anos de 2001 a 2020.

\begin{tabular}{cccccccccc}
\hline Ano & \multicolumn{2}{c}{ Positivo } & \multicolumn{2}{c}{ Negativo } & \multicolumn{2}{c}{ Em andamento } & \multicolumn{2}{c}{ Não realizado } & Total \\
\hline & $\mathbf{n}$ & $\mathbf{\%}$ & $\mathbf{n}$ & $\mathbf{\%}$ & $\mathbf{n}$ & $\boldsymbol{\%}$ & $\mathbf{n}$ & $\mathbf{\%}$ & \\
2001 & 7 & 2,75 & 23 & 9,05 & 17 & 6,7 & 207 & 81,50 & 254 \\
2002 & 6 & 2,26 & 15 & 5,63 & 21 & 7,9 & 224 & 84,21 & 266 \\
2003 & 8 & 3,72 & 31 & 14,41 & 26 & 12,10 & 150 & 69,77 & 215 \\
2004 & 3 & 1,36 & 42 & 19,17 & 26 & 11,9 & 148 & 67,57 & 219 \\
2005 & 9 & 4,26 & 54 & 25,6 & 11 & 5,21 & 137 & 64,93 & 211 \\
2006 & 12 & 5,06 & 91 & 38,40 & 12 & 5,06 & 122 & 51,48 & 237 \\
2007 & 7 & 3,36 & 84 & 40,40 & 5 & 2,40 & 112 & 53,84 & 208 \\
2008 & 3 & 1,70 & 91 & 51,41 & 4 & 2,25 & 79 & 44,64 & 177 \\
2009 & 14 & 7 & 96 & 48 & 3 & 1,5 & 87 & 43,5 & 200 \\
2010 & 11 & 6 & 79 & 42,93 & 5 & 2,71 & 89 & 48,36 & 184 \\
2011 & 8 & 4,18 & 97 & 50,8 & 8 & 4,18 & 78 & 40,84 & 191 \\
2012 & 9 & 4,81 & 85 & 45,45 & 11 & 5,89 & 82 & 43,85 & 187 \\
2013 & 14 & 7,45 & 115 & 61,17 & 9 & 4,78 & 50 & 26,60 & 188 \\
2014 & 13 & 7,83 & 130 & 78,31 & - & 0 & 23 & 13,86 & 166 \\
2015 & 11 & 7,05 & 133 & 85,26 & 1 & 0,64 & 11 & 7,05 & 156 \\
2016 & 13 & 7,56 & 125 & 72,67 & 1 & 0,58 & 33 & 19,19 & 172 \\
2017 & 13 & 8,17 & 115 & 72,33 & 2 & 1,26 & 29 & 18,24 & 159 \\
2018 & 11 & 5,58 & 178 & 90,36 & - & 0 & 8 & 4,06 & 197 \\
2019 & 11 & 5,28 & 189 & 90,87 & - & 0 & 8 & 3,85 & 208 \\
2020 & 16 & 8,90 & 132 & 73,33 & 6 & 3,33 & 26 & 14,44 & 180 \\
\hline
\end{tabular}

Fonte: Autores (2021).

Observa-se que o percentual de testagem para HIV nos casos de TB aumentou ao longo dos 20 anos e foi inversamente proporcional a quantidade de pacientes não testados. Em 2001 foram testados 18,50\% dos casos de TB para HIV e em 2020 a testagem foi de $85,55 \%$ dos casos. Consequentemente, os casos de coinfecção HIV/TB aumentaram de 2,75\% em 2001 para $8,90 \%$ em 2020.

A Tabela 3 evidencia as varáveis que caracterizam o aspecto sociodemográfico e a clínica dos casos novos de TB coinfectados por HIV que residem no Estado do Tocantins.

Tabela 3 - Caracterização sociodemográfica e clínica de casos novos de tuberculose e coinfecção HIV residentes no estado do Tocantins, Brasil, nos anos 2001 a 2020.

\begin{tabular}{ccc}
\hline Variáveis & $\mathbf{N}(\mathbf{1 9 9})$ & $\%$ \\
\hline Sexo & 152 & 76,39 \\
Masculino & 47 & 23,61 \\
Feminino & & 1,50 \\
Faixa etária & 3 & 0 \\
< 5 anos & - & 55,80 \\
5 a 19 & 111 & 36,20 \\
20 a 39 & 72 & 6,50 \\
40 a 59 & 13 & \\
60 mais & & 68,87 \\
Etnia & 137 & 13,06 \\
Parda & 26 & 8,54 \\
Branca & 17 & 0,50 \\
Preta & 1 & 5,52 \\
Amarela & 11 & 3,51 \\
Indígena & 7 & 6,53 \\
Não preenchido & & 21,10 \\
Escolaridade/anos de estudo & 13 & 27,63 \\
Analfabeto & 42 &
\end{tabular}




\begin{tabular}{ccc}
\hline 9 a 12 & 25 & 12,59 \\
+12 anos & 12 & 6,03 \\
Não se aplica & 3 & 1,50 \\
Não preenchidos & 49 & 24,62 \\
Regiões de saúde & & \\
Capim Dourado & 72 & 36,20 \\
Médio Norte Araguaia & 35 & 17,60 \\
Bico do Papagaio & 23 & 11,55 \\
Cantão & 19 & 9,54 \\
Amor Perfeito & 19 & 9,54 \\
Ilha do Bananal & 14 & 7,05 \\
Cerrado Tocantins Araguaia & 10 & 5,01 \\
Sudeste & 7 & 3,51 \\
Forma Clínica & 121 & \\
Pulmonar & 68 & 60,80 \\
Extrapulmonar & 10 & 34,17 \\
Pulmonar + extrapulmonar & & 5,03 \\
\hline
\end{tabular}

Fonte: Autores (2021).

Nesse sentido, os casos de coinfecção de HIV/TB predominaram no sexo masculino (152/76,39\%), faixa etária de 2039 anos (111/55,80\%), pessoas pardas (137/68,87\%), com 1 a 8 anos de estudos (97/48,73\%), sendo que o não preenchimento da escolaridade foi de $24,62 \%$ dos casos. A região de saúde Capim Dourado foi a que mais notificou a coinfecção HIV/TB (72/36,20\%), seguida da região de saúde Médio Norte Araguaia (35/17,60\%). A região de saúde Sudeste foi a que menos registrou a coinfecção HIV/TB (7/3,51\%). Predominou a forma clínica pulmonar (121/60,80\%), seguida da forma clínica extrapulmonar $(68 / 34,17 \%)$.

A Tabela 4 exibe a situação de encerramento dos casos novos de TB coinfectados por HIV no período do estudo.

Tabela 4 - Frequência da situação de encerramento de casos novos de tuberculose com coinfecção HIV residentes no estado do Tocantins, Brasil, nos anos de 2001 a 2020.

\begin{tabular}{ccc}
\hline Variáveis & N (199) & \% \\
\hline Situação de encerramento & & 45,22 \\
Cura & 90 & 10,05 \\
Abandono & 20 & 3,01 \\
Óbito por tuberculose & 6 & 22,11 \\
Óbito por outras causas & 44 & 15,10 \\
Transferência & 30 & 1,00 \\
Tuberculose RM & 2 & 3,51 \\
Não preenchido & 7 & \\
\hline
\end{tabular}

*RM= tuberculose resistente à medicação. Fonte: Autores (2021).

Verifica-se que a proporção de encerramento dos casos com coinfecção HIV/TB devido a cura foi de 45,22\%, abandono do tratamento $10,05 \%$, transferência $15,10 \%$, óbitos por outras causas $22,11 \%$ e óbitos por tuberculose $3,01 \%$.

\section{Discussão}

Este estudo identificou uma prevalência de 5\% da coinfecção por HIV entre os casos de TB e um crescimento importante nesse indicador ao longo dos 20 anos na avaliação do estudo no estado do Tocantins. O percentual de testagem para HIV nos casos de TB aumentou ao longo dos 20 anos e foi inversamente proporcional a quantidade de pacientes não testados. O estudo também mostra a vulnerabilidade social entre os casos de coinfecção de HIV/TB, pois predominaram jovens adultos de cor parda, com menos de oito anos de estudo e homens. A proporção de cura foi muito baixa e a região de saúde mais populosa do estado (Capim Dourado) registrou a maior frequência de casos de coinfecção HIV/TB. 
É fato que a tuberculose (TB) e as coinfecções por HIV representam um fardo imenso para os sistemas de saúde e representam desafios diagnósticos e terapêuticos específicos nos diferentes territórios. A infecção pelo HIV é o fator de risco conhecido mais poderoso que predispõe à infecção pelo Mycobacterium tuberculosis e à progressão para doença ativa, o que aumenta em 20 vezes o risco de reativação latente da TB. A tuberculose também é a causa mais comum de morte relacionada à AIDS. Assim, M. tuberculosis e o HIV agem em sinergia, acelerando o declínio das funções imunológicas e levando à morte subsequente se não for tratado (Pawlowski, 2012; Nhandara, 2021).

Na dupla interação HIV / M. tuberculosis não apenas o HIV afeta a progressão da TB, mas a infecção por $M$. tuberculosis também tem um impacto negativo na resposta imunológica ao HIV, acelerando a progressão da infecção pelo HIV para a AIDS (Whalen, 1995; Bruchfeld, 2015).

No Tocantins, a prevalência de 5\% de coinfecção por HIV entre os casos de TB corrobora com a literatura, pois o risco ao longo da vida de desenvolver TB ativa em adultos imunocompetentes é estimado em 5\% a 10\% durante a vida, mas em indivíduos HIV-positivos esse risco aumenta para 5\% a 15\% anualmente (Aaron, 2004).

O aumento na prevalência da coinfecção TB-HIV no período analisado evidencia falhas dos programas no controle da TB e, sobretudo, do HIV, relacionado à introdução tardia da terapia antirretroviral (TARV) e dificuldade de acesso aos serviços de referência (Pinto, 2017; Cavalin, 2020).

Por outro lado, a ampliação da testagem no estado do Tocantins nos últimos anos sinaliza uma melhor organização dos serviços de saúde para a assistência à TB e ao HIV/aids, bem como o aprimoramento das ações de controle da TB e do HIV/aids.

Ademais, o aumento dos casos positivos de coinfecção HIV/TB pode ser decorrente da implementação de estratégias de combate à doença nos últimos anos, bem como melhorias nas condições de atendimento de saúde e maior acesso populacional aos cuidados continuados pela Atenção Primária à Saúde (APS). Com exceção dos três últimos anos, o Ministério da Saúde realizou ações de visibilidade política e articulação multissetorial no controle da doença. Ademais, a APS apresenta boa cobertura em vários estados e apresenta cobertura populacional por Equipes de Saúde da Família (EqSF), entre eles estão o Piauí (90,7\%), Tocantins (89,7\%), Santa Catarina (87,2\%), Paraíba (86,7\%) e Sergipe (82,6\%). Outro fator preponderante para o aumento dos casos foi o avanço tecnológico dos testes sorológicos e maior cobertura do tratamento (Giovanella, 2021).

O aumento de cobertura da APS no país tem sido considerado como uma importante ferramenta para a melhoria dos indicadores de TB nos municípios brasileiros. No entanto, o processo de descentralização das ações de controle da TB para a APS tem ocorrido de modo verticalizado. Essa verticalização é um fator que deve ser corrigida em áreas com boa cobertura de APS, pois o controle da coinfecção HIV/TB com redução do coeficiente de incidência de tuberculose em $95 \%$ até o ano de 2035 só é possível com a descentralização (Silva, Menezes \& Oliveira, 2014).

Adicionalmente, a Organização Mundial da Saúde (OMS) refere que nos últimos anos observou-se considerável progresso na realização de testagem sorológica para o HIV em pacientes com diagnóstico confirmado de tuberculose nos diferentes países, em especial Brasil (Chakaya, 2021).

Nos aspectos sociodemográficos, o predomínio de casos em pessoas com baixa escolaridade, sexo masculino, e cor parda refletem as vulnerabilidades destes indivíduos, dificultando, assim, o seu acesso aos serviços de saúde, o que corrobora com a literatura (Velásquez, 2015; Cavalin, 2020; Boletim Epidemiológico HIV/TB, 2020). A distribuição de idade na população foi fortemente inclinada para os grupos de idade mais jovem, e estima-se que mais da metade da população de 15-49 anos esteja infectada com M. tuberculosis e desenvolva TB ativa com mais frequência, fato constatado na população desse estudo. Esses jovens adultos também estão em maior risco de infecção por HIV, resultando em epidemias sobrepostas nessa faixa etária (Bruchfeld, 2015). 
O predomínio do sexo masculino seguiu o padrão nacional conforme os dados do último boletim epidemiológico de 2020. Essa prevalência pode ser justificada pelo aumento na notificação de casos de AIDS no sexo masculino (Boletim Epidemiológico HIV/TB, 2020). O predomínio da carga fatal e não fatal pela TB no sexo masculino pode ser explicado pela maior exposição ao bacilo nas atividades laborais e pela frequência de comportamentos de risco para a doença nesse gênero, como maior consumo de bebidas alcoólicas, tabagismo e uso de drogas. Além disso, destacou-se a diferença de sexo no risco de TB devido a comportamentos de cuidado à saúde, como menor autocuidado, menor demanda por assistência à saúde e menor adesão e maior abandono e perda de seguimento ao tratamento de TB no sexo masculino (Martins, 2017; Ledesma, 2021). Apesar da ênfase na equidade no acesso ao diagnóstico e tratamento da tuberculose pela Estratégia da OMS para Acabar com a TB, as diferenças na carga da tuberculose por sexo têm recebido pouca atenção. As evidências disponíveis indicam que os homens são menos propensos a buscar ou ter acesso aos cuidados de tuberculose do que as mulheres (Ledesma, 2021).

A forma clínica pulmonar, presente em $60 \%$ dos casos com coinfecção HIV/TB, está relacionada ao maior risco de transmissão, intrinsecamente ligado por aspectos como a exposição ambiental, condições precárias de moradia e alimentação (Oliveira, 2017; Cavalin, 2020). Por outro lado, a forma clínica extrapulmonar apresentou elevada magnitude, com quase $35 \%$ dos casos. Isso reporta o comprometimento imunológico importante e AIDS avançada nas formas extrapulmonares da TB (Velásquez, 2015; Cavalin, 2020). Contudo, o tratamento adequado com o uso da TARV promove a manutenção da competência do sistema imune e está associada à diminuição do coeficiente incidência de TB (Kwan, 2011).

A região de saúde do estado com maior taxa de coinfecção HIV/TB foi a Capim Dourado, provavelmente pela maior concentração populacional nessa área. Nessa região está localizada Palmas, a capital do estado. Certamente, por possuir maior densidade demográfica, especialmente na capital e áreas metropolitanas apresentam maior aglomeração urbana, seja de moradores, trabalhadores, unidades de saúde e usuários do transporte público, o que acarreta maior fluxo e encontro de pessoas, além de maior possibilidade de transmissão de doenças como a TB. Esse fato deve levar em consideração que ao formular medidas de prevenção e controle para a coinfecção TB-HIV, há que se considerar os aspectos determinantes da transmissão e do controle do HIV nos centros urbanos mais populosos (Cavalin, 2020; Chakaya, 2021). Compreender os aspectos que determinam a concentração dos casos de coinfecção TB-HIV nas diferentes regiões de saúde do estado do Tocantins pode favorecer as medidas de prevenção e controle da doença em áreas de maior risco e facilitar a organização dos programas de controle para o enfrentamento do problema e oferecer assistência às pessoas com TB / HIV/AIDS.

Quanto aos aspectos relacionados ao diagnóstico da TB e ao encerramento do tratamento, com destaque para a grande parcela de diagnósticos realizados em hospitais e as elevadas taxas de abandono e óbito, refletem as dificuldades em se efetivar a busca ativa de casos, o diagnóstico precoce e o tratamento efetivo da coinfecção TB-HIV no Tocantins (Cavalin, 2020).

As limitações desse estudo estão relacionadas à análise de dados secundários, o que pode estar sujeito às falhas de preenchimento e/ou incompletude das informações, além da falta de informações que podem ser importantes para as análises de interesse, no estado do Tocantins. Porém, diante da carência de estudos que analisem dados epidemiológicos da coinfecção HIV/TB no estado, os resultados aqui apresentados são relevantes para o subsídio de ações e na tomada de decisão dos órgãos públicos estatais e municipais na concepção de políticas públicas considerando a realidade local.

\section{Conclusão}

$\mathrm{O}$ aumento de casos de tuberculose coinfectados com HIV, a gravidade dos casos na forma clínica extrapulmonar, o baixo percentual de cura e alta carga da letalidade representam os desafios que os programas de controle precisam superar para o manejo clínico da tuberculose associada ao HIV no estado do Tocantins. O principal desafio inclui a integração de um 
tratamento eficaz de tuberculose associado ao uso de terapia antirretroviral para a prevenção de comorbidades relacionadas ao HIV.

Ademais, pode-se perceber que o presente estudo compreende um problema de saúde que necessita de pesquisas constantes que apontem e melhorem o diagnóstico, tratamento e seguimento das doenças citadas. Assim como no Tocantins, em âmbito nacional, os estudos dessas patologias também devem ser ampliados, no intuito de promover melhorias substanciais do presente cenário.

\section{Referências}

Aaron L, Saadoun D, Calatroni I et al. (2004). Tuberculosis in HIV-infected patients: a comprehensive review. Clinical Microbiology and Infection, 10(5), 388-398.

Brasil, Ministério da Saúde. (2019). Situação epidemiológica - dados. Portal da Saúde. http://portalarquivos.saude.gov.br/images/pdf/2019/fever eiro/11/Tabela\%20Geral_1208.pdf.

Brasil, Ministério da Saúde. (2020). Gabinete do Ministro. Portaria n 264, de 17 de fevereiro de 2020. Brasília, 2020. https://www.in.gov.br/en/web/dou//portaria-n-264-de-17-de-fevereiro-de-2020-244043656.

Brasil, Ministério da Saúde. (2020). Programa Nacional de Controle da Tuberculose - PNCT. 2020.

Brasil, Ministério da Saúde. (2021). Panorama Epidemiológico da coinfecção TB-HIV no Brasil 2020. Brasília. http://www.aids.gov.br/ptbr/pub/2021/panorama-epidemiologico-da-coinfeccao-tb-hiv-no-brasil-2020.

Brasil. (2017). Brasil Livre da Tuberculose: Plano Nacional pelo Fim da Tuberculose como Problema de Saúde Pública. Ministério da Saúde, Secretaria de Vigilância em Saúde, Departamento de Vigilância das Doenças Transmissíveis. - Brasília: Ministério da Saúde.

Brasil. (2019). Manual de recomendações para o controle da tuberculose no Brasil, $2^{\mathrm{a}}$ Edição. Tratamento da tuberculose em condições especiais. Ministério da Saúde, Secretaria De Vigilância em Saúde. Departamento de Vigilância das Doenças Transmissíveis. http://www.aids.gov.br/pt-br/pub/2019/manual-derecomendacoes-para-o-controle-da-tuberculose-no-brasil.

Brasil. (2021). Indicador $\mathrm{n}^{\circ}$ 17. Proporção de cura dos casos novos de tuberculose pulmonar bacilífera por local de residência. http://idsus.saude.gov.br/ficha17s.html.

Brasil. Ministério da Saúde. (2021). DATASUS. http://datasus.saude.gov.br/informacoes-de-saude-tabnet/.

Brasil. Ministério da Saúde. (2021). Guia de Vigilância em Saúde. Brasília: Ministério da Saúde. http://portalarquivos.saude.gov.br/images/pdf/201 7/outubro/06/ Volume-Unico-2017.pdf.

Bruchfeld, J., Correia-Neves, M., \& Källenius, G. (2015). Tuberculosis and HIV coinfection. Cold Spring Harbor perspectives in medicine, 5(7), a017871.

Campos, V. R. V., \& de Carvalho, J. A. (2021). Perfil epidemiológico da população privada de liberdade notificada com tuberculose no Tocantins de 2008 a 2018. Revista de Patologia do Tocantins, 8(1), 11-16.

Cavalin, R. F., Pellini, A. C. G., Lemos, R. R. G. D., \& Sato, A. P. S. (2020). Coinfecção TB-HIV: distribuição espacial e temporal na maior metrópole brasileira. Revista de Saúde Pública, 54

Chakaya, J. et al. (2021). Global Tuberculosis Report 2020-Reflections on the Global TB burden, treatment and prevention efforts. International Journal of Infectious Diseases.

Costa, M.F.L. \& Barreto, S.M. (2003). Tipos de estudos epidemiológicos: conceitos básicos e aplicações na área do envelhecimento. Epidemiologia e Serviços de Saúde, 12(4), 189-201. https://dx.doi.org/10.5123/S1679-49742003000400003

da Cruz Silva, P. H., Vaz, G. P., Bitencourt, E. L., \& da Costa, S. B. (2020). Análise comparativa do perfil epidemiológico da tuberculose no estado do Tocantins e região norte do Brasil entre 2009 e 2019. Revista de Patologia do Tocantins, 7(1), 3-9.

GIL, A. (1999). Métodos e Técnicas de Pesquisa Social. (5a ed.), editora Atlas.

Giovanella, L. et al. (2021). Cobertura da Estratégia Saúde da Família no Brasil: o que nos mostram as Pesquisas Nacionais de Saúde 2013 e 2019. Ciência \& Saúde Coletiva, 26, 2543-2556.

IBGE. (2011). Estimativas de população - População Residente - Estimativas para o TCU - Tocantins, acesso DATASUS.

Kwan, C. K., \& Ernst, J. D. (2011). HIV and tuberculosis: a deadly human syndemic. Clinical microbiology reviews, 24(2), 351-376.

Ledesma, J. R. et al. (2021). Global, regional, and national sex differences in the global burden of tuberculosis by HIV status, 1990-2019: results from the Global Burden of Disease Study 2019. The Lancet Infectious Diseases.

Magno, E. D. S. et al. (2017). Fatores associados à coinfecção tuberculose e HIV: o que apontam os dados de notificação do Estado do Amazonas, Brasil, 2001-2012. Cadernos de Saúde Pública, 33, e00019315. 
Research, Society and Development, v. 10, n. 14, e278101422279, 2021 (CC BY 4.0) | ISSN 2525-3409 | DOI: http://dx.doi.org/10.33448/rsd-v10i14.22279

Marconi, M. D. A., \& Lakatos, E. M. (2003). Fundamentos de metodologia científica. (5a ed.), Atlas.

Martins-Melo, F. R. et al. (2020). A carga da tuberculose e fatores de risco atribuíveis no Brasil, 1990-2017: resultados do Global Burden of Disease Study 2017. Popul Health Metrics, 18, 10. https://doi.org/10.1186/s12963-020-00203-6.

Nhandara, R. B. C., Ayele, B. T., Sigwadhi, L. N., Ozougwu, L. U., \& Nyasulu, P. S. (2020). Determinants of adherence to clinic appointments among tuberculosis and HIV co-infected individuals attending care at Helen Joseph Hospital, Johannesburg, South Africa. The Pan African Medical Journal, 37.

Oliveira, A. A. V. et al. (2017). The access of the homeless persons with tuberculosis to the health care: an integrative review. Int Arch Med, 10(10.3823), 2384 .

Pawlowski, A., Jansson, M., Sköld, M., Rottenberg, M. E., \& Källenius, G. (2012). Tuberculosis and HIV co-infection. PLoS pathogens, 8(2), e1002464.

Pinto, P. F. P. S. et al. (2017). Epidemiological profile of tuberculosis in São Paulo municipality from 2006 to 2013 . Revista Brasileira de Epidemiologia, 20, $549-557$.

Ribas, J. L. C., Amoreli, J., Cardoso, M. C. R., Schoepfer, H. P., Borges, M. P., \& Monteiro, L. D. (2021). Tendência temporal da tuberculose na população privada de Liberdade no Estado do Tocantins. Research, Society and Development, 10(6), e7910615400-e7910615400.

Richardson, R. J., Peres, J. A. S., Wanderley, J. C. V., Correia, L. M., \& Peres, M. H. M. (1999). Pesquisa social: métodos e técnicas. São Paulo: Editora Atlas. Secretaria de Saúde. (2018). Regionalização da Saúde Tocantins - História e Mapas, 2018. Governo do Tocantins. https://www.to.gov.br/saude/regionalizacaoda-saude-tocantins-historia-e-mapas/468kh0pycna2.

Silva, H.O., Gonçalves, M. L. C. (2009). Coinfecção tuberculose e hiv nas capitais brasileiras: observações a partir dos dados do sistema de informação de agravos de notificação. Revista Brasileira em Promoção da Saúde. 22(3), 172-178. https://www.redalyc.org/articulo.oa?id=40812007007.

Silva, M. A. P. D., Menezes, R. C. E. D., Oliveira, M. A. A., Longo-Silva, G., \& Asakura, L. (2014). Atenção Básica em Alagoas: expansão da Estratégia Saúde da Família, do Nasf e do componente alimentação/nutrição. Saúde em Debate, 38, 720-732.

Velásquez, G. E., et al. (2015). Impact of HIV on mortality among patients treated for tuberculosis in Lima, Peru: a prospective cohort study. BMC infectious diseases, 16(1), 1-11.

Whalen, C. et al. (1995). Accelerated course of human immunodeficiency virus infection after tuberculosis. American journal of respiratory and critical care medicine. 151(1), 29-135.

World Health Organization. (2008). Global tuberculosis control: surviellance, planning, financing: WHO report 2008. www.who.int/tb/publications/global_report/2008/pdf/fullrereport.pdf. 